\title{
Complete mitochondrial genomes reveal phylogeny relationship and evolutionary history of the family Felidae
}

\author{
W.Q. Zhang and M.H. Zhang \\ College of Wildlife Resources, Northeast Forestry University, Harbin, \\ Heilongjiang Province, China \\ Corresponding author: M.H. Zhang \\ E-mail: zhangminghai2004@126.com
}

Genet. Mol. Res. 12 (3): 3256-3262 (2013)

Received July 31, 2012

Accepted October 8, 2012

Published September 3, 2013

DOI http://dx.doi.org/10.4238/2013.September.3.1

\begin{abstract}
Many mitochondrial DNA sequences are used to estimate phylogenetic relationships among animal taxa and perform molecular phylogenetic evolution analysis. With the continuous development of sequencing technology, numerous mitochondrial sequences have been released in public databases, especially complete mitochondrial DNA sequences. Using multiple sequences is better than using single sequences for phylogenetic analysis of animals because multiple sequences have sufficient information for evolutionary process reconstruction. Therefore, we performed phylogenetic analyses of 14 species of Felidae based on complete mitochondrial genome sequences, with Canis familiaris as an outgroup, using neighbor joining, maximum likelihood, maximum parsimony, and Bayesian inference methods. The consensus phylogenetic trees supported the monophyly of Felidae, and the family could be divided into 2 subfamilies, Felinae and Pantherinae. The genus Panthera and species tigris were also studied in detail. Meanwhile, the divergence of this family was estimated by phylogenetic analysis using the Bayesian method with a relaxed molecular clock, and the results shown were consistent with previous studies. In summary, the evolution of Felidae was reconstructed by phylogenetic analysis
\end{abstract}


based on mitochondrial genome sequences. The described method may be broadly applicable for phylogenetic analyses of anima taxa.

Key words: Felidae; Mitochondrial genome; Bayesian method; Evolutionary analysis

\section{INTRODUCTION}

Felidae is the biological family of the cat, and a member of this family is called a felid. The felid family consists of 2 major subfamilies: Pantherinae (which includes the tiger, the lion, the jaguar, and the leopard) and Felinae (which includes the cougar, the cheetah, the lynxes, the ocelot, and the domestic cat) (Johnson et al., 2006; Christiansen, 2008). Felidae includes more than 41 living species of felids worldwide, and all of these species have descended from the same ancestor. The ancestor likely lived in Asia during the Oligocene and then spread across continents through land bridges. Modern-day cat species originated during the late Miocene and evolved into the strictest carnivores of the 13 terrestrial families of the order Carnivora (Glazko and Nei, 2003; Yang and Yoder, 2003; Yu et al., 2004). However, because of the rapid and recent speciation events, few distinguishing dental and skeletal characteristics, incidents of parallel evolution, and the incomplete fossil record, determination of the evolutionary history and divergence of Felidae members is challenging (Weissengruber et al., 2002).

Traditionally, the evolutionary history of Felidae has been determined by morphological methods, e.g., skull and mandible shape (Christiansen, 2008; Mazák et al., 2011). Since the development of molecular biology techniques, many molecular approaches have been used for classification of Felidae; for example, comparative cytogenetics, albumin immunological distance determination, DNA-DNA hybridization, isoenzyme identification by 2-dimensional gel electrophoresis, differential segregation of integrated retroviral sequences, sex chromosome-linked gene identification, and chemical signal determination. More recently, some molecules were introduced to delimit the phylogenetic tree of Felidae, such as introns, SINEs, CYP2D6, D-loops, and other mitochondrial genes (Johnson and O'Brien, 1997; Pecon-Slattery et al., 2004; Yu and Zhang, 2005; Zhang et al., 2006). However, these genes are biased, and the phylogenetic trees may not be suitable, especially for large groups.

Mitochondrial DNA (mtDNA) is a small circular molecule of $15-20 \mathrm{~kb}$ and is conserved in vertebrates. mtDNA is highly variable in structure, gene content, organization, and mode of expression in the different organisms (Grande et al., 2008; Flegontov et al., 2011). Therefore, mtDNA is regarded as the marker of choice for the reconstruction of phylogenetic relationships among vertebrates, population genetics, and molecular evolution analysis. The 37 genes in the mitochondrial genome include 13 protein-coding genes, 22 tRNA-encoding genes, 2 rRNA-encoding genes, and a regulatory region (D-loop). Because the mitochondrial genome has several unique characteristics, including its small size, abundance in animal tissues, strict orthology of encoded genes, and uniparental inheritance, mtDNA is a reliable and easy-to-use phylogenetic marker (Kan et al., 2010; Krajewski et al., 2010; Li et al., 2010; Md-Zain et al., 2010; Morin et al., 2010). Many phylogenetic trees have been constructed for animals. Most of these trees have been reconstructed from single genes or gene elements, e.g., the cyb gene and D-loop region. However, the evolutionary information encoded in single genes or genetic elements is limited, and the resulting phylogenetic analysis is thus biased. 
mtDNA sequences are rapidly accumulating in public databases, and there are numerous mitochondrial genomes in animal available in GenBank/EMBL/DDBJ. Therefore, more scientists study the evolution of organisms based on mitochondrial genome sequences. In Felidae, approximately 14 mitochondrial genomes from 2 subfamilies have been sequenced, and the mtDNA sequences are publicly available, enabling phylogenetic analysis.

In this study, we present phylogenetic analyses based on Felidae mitochondrial genomes retrieved from GenBank. We used molecular data to examine hypotheses of the evolution and classification of Felidae, focusing on the evolutionary relationship of subfamilies of the genus Panthera.

\section{MATERIAL AND METHODS}

\section{Phylogeny and evolutionary analysis of Felidae}

All Felidae mitochondrial genome sequences were downloaded from the NCBI website. The accession numbers of the 14 species of Felidae are listed in Table 1 (Wei et al., 2011; Zhang et al., 2011; Kitpipit et al., 2012). All protein-coding regions, tRNA-encoding regions, and rRNAencoding regions were identified by homology-based annotation. The mitochondrial genome sequence of Canis familiaris was also downloaded for use as an outgroup, and all sequences were aligned using ClustalW2 with manual adjustment (Larkin et al., 2007). The multiple sequence alignment of 15 genomes was the input into MEGA4 to perform evolutionary analysis (Ronquist and Huelsenbeck, 2003; Tamura et al., 2007). Neighbor joining (NJ) analysis with the bootstrap test (1000 replicates) was performed in MEGA using pairwise deletion for gaps and missing data. However, due to the lack of data partitioning of the GTR model in MEGA, the non-partitioned dataset and the best available Tamura-Nei model were used for the NJ reconstruction.

Table 1. Species and accession numbers of mitochondria genome sequences used in this study.

\begin{tabular}{ll}
\hline Species & Accession No. \\
\hline Panthera tigris altaica & HM185182 \\
P. tigris sumatrae & JF357970 \\
P. tigris tigris & JF357968 \\
P. tigris corbetti & JF357971 \\
P. tigris amoyensis & NC_014770 \\
P. uncia & EF551004 \\
P. leo persica & JQ904290 \\
P. pardus & NC_010641 \\
Neofelis nebulosa & NC_008450 \\
Prionailurus bengalensis euptilura & NC_016189 \\
Felis catus & FCU20753 \\
Puma concolor & NC_016470 \\
Acinonyx jubatus & NC_005212 \\
Lynx rufus & NC_014456 \\
Canis familiaris & U96639 \\
\hline
\end{tabular}

A Bayesian approach, implemented in MrBayes 3.1.2, was used to analyze nucleotide and amino acid datasets using the GTR model with a gamma-distributed rate variation (Ronquist and Huelsenbeck, 2003). Four Metropolis-coupled Markov-chain Monte Carlo (MCMC) analyses were run twice for 10,000,000 generations, with sampling every 100 generations. After the first 2500 trees $(25 \%)$ had been discarded as burn-in, the remaining 7500 
sampled trees were used to estimate the $50 \%$ majority rule consensus tree and the Bayesian posterior probabilities. The results were visualized using Mesquite.

\section{Estimation of divergence times in Felidae}

Two calibration points for the Felidae family were used for the analysis (Johnson et al., 2006). Calibration points enable the assignment of ages to nodes in the phylogenetic trees; the age of the oldest fossil approximates the minimum age of a node. The divergence times within the Felidae family were estimated using the Bayesian method implemented in BEAST, using a relaxed molecular clock (Drummond and Rambaut, 2007). BEAST was configured as follows: the substitution models were implemented using the $G T R+I+\Gamma$ model, and 10,000,000 generations of MCMC steps were sampled every 5000 generations during two independent runs. The log output files were combined using LogCombiner 1.5.3, and the effective sample size values $(>350)$ were adequate for all parameters. The convergence was assessed in Tracer 1.5, and the first 1000 samples $(10,000,000$ generations) were excluded as burn-in. The chronological tree files were analyzed and visualized using TreeAnnotator 1.5.3 and FigTree 1.3.1.

\section{RESULTS}

\section{Monophyly of Felidae}

The NJ and Bayesian phylogenetic trees, based on mitochondrial genome sequences, have similar topology, as shown in Figure 1. The 2 subfamilies, Felinae and Pantherinae, are shown from bottom to top. The monophyly of Felidae was well supported, with a bootstrap value of $100 \%$ in NJ and a posterior probability of 1.00 in Bayesian. The phylogenetic tree showed that Felinae and Pantherinae diverged nearly simultaneously. The phylogenetic tree showed not only the phylogeny of the family but also the monophyly of the subfamilies, e.g., the subfamily Pantherinae, and the phylogenetic tree provided evolutionary information about Neofelis nebulosa, Panthera uncia, P. leo persica, P. pardus, and P. tigris. Furthermore, the phylogenetic tree could be used to determine classification of species, for example, $P$. tigris. The phylogenetic trees showed that $P$. tigris altaica diverged first, followed by $P$. $t$. amoyensis, followed by $P$. $t$. tigris, $P$. t. sumatrae, and $P$. t. corbetti, consistent with previous findings (Weissengruber et al., 2002; Luo et al., 2004; Mazák et al., 2011). Felinae is divided into 2 main clades, corresponding to small- to medium-sized cats, including Felis catus and Prionailurus bengalensis, and larger animals, including Puma concolor, Acinonyx jubatus, and Lynx rufus.

\section{Estimation of divergence times in Felidae}

From 2 calibration points, the divergence times were estimated. The results are illustrated in Figure 2. Felidae emerged 14 mya, and after divergence, subfamilies Pantherinae and Felinae emerged 9.96 and 9.13 mya, respectively. In subfamily Pantherinae, N. nebulosa emerged first, followed by P. tigris, P. uncia, P. leo persica, and by P. pardus, at 7.07, 5.21, and 3.72 mya, respectively. P. tigris diverged within the last 2 million years, and Amur (Siberian

tiger, P. t. altaica) emerged 2.38 mya; the Amoy (South China tiger, P. t. amoyensis) followed 


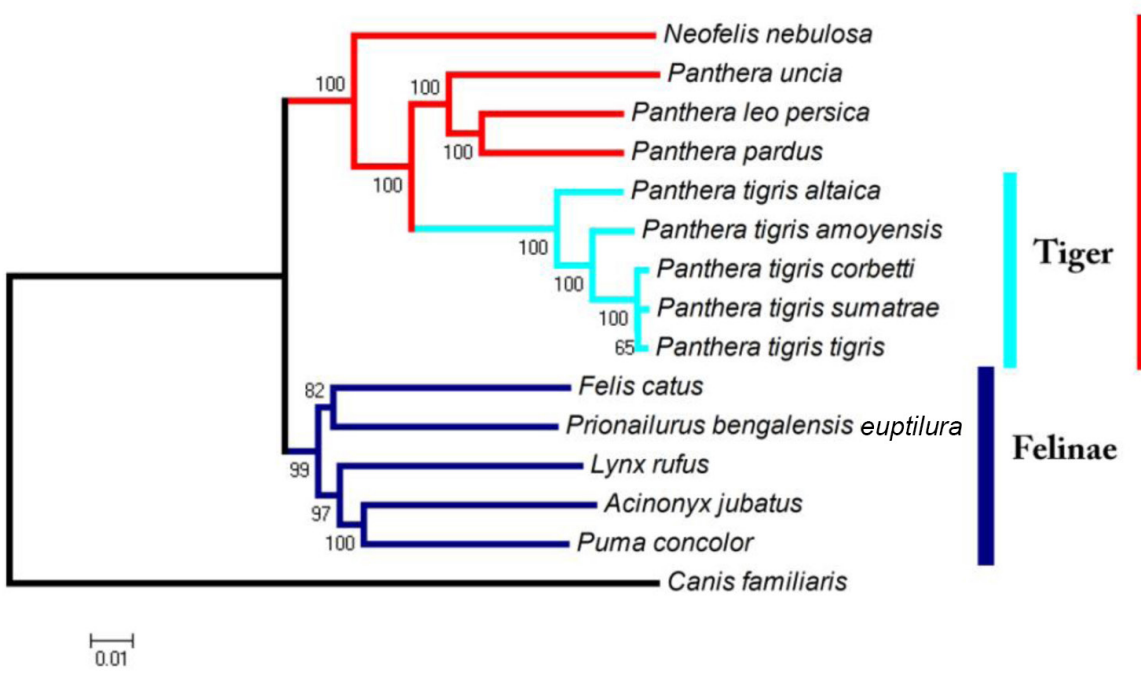

Pantherinae

Figure 1. Phylogenetic tree of the relationships among family Felidae based on whole mitochondrium genomes. Canis familiaris served as outgroup.

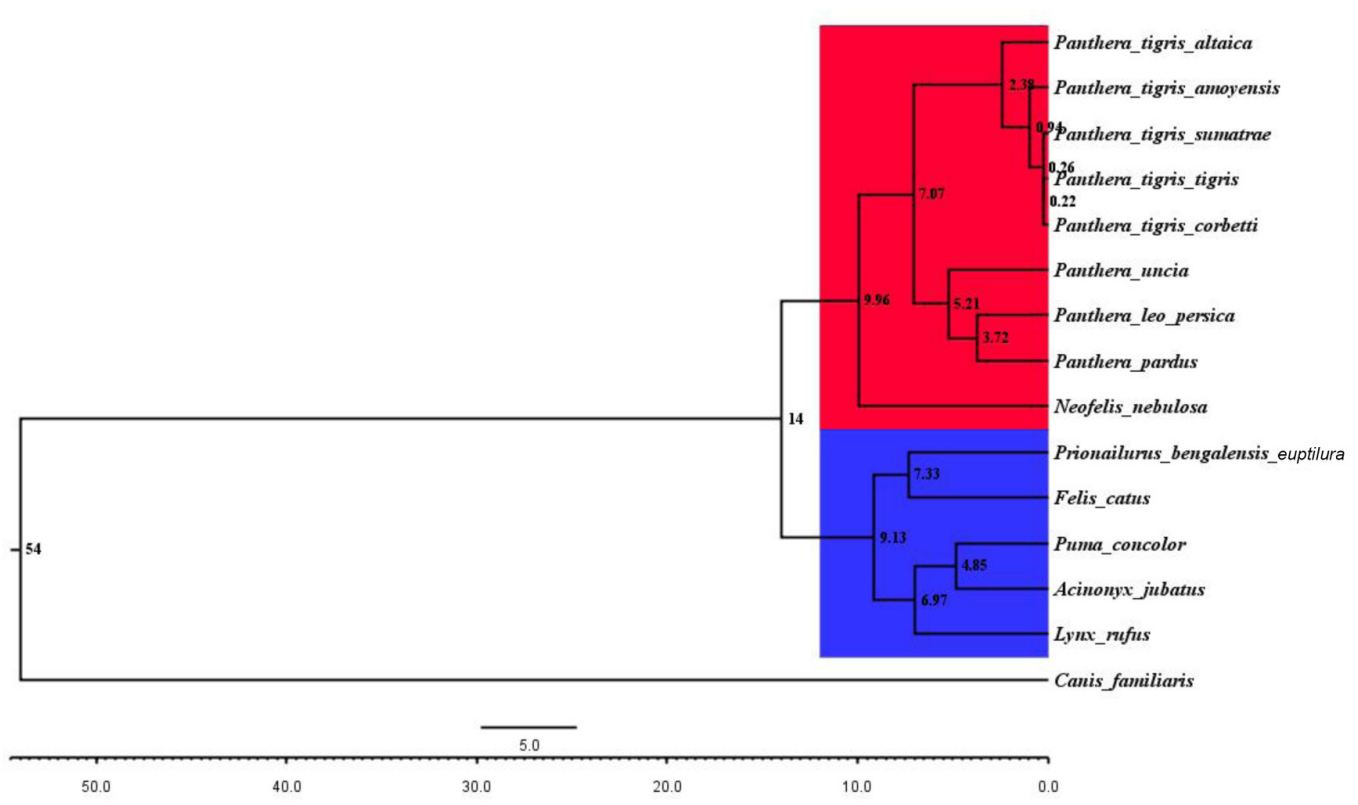

Figure 2. Phylogenetic tree of family Felidae and outgroup Canis familiaris based on the whole mitochondria genomes. The divergence times were estimated by Bayesian method, and they are shown on the labels of nodes. 
(approximately 0.94 mya), with the Indochinese tiger (P. t. corbetti), Sumatra tiger (P. t. sumatra), and Bengal tiger (P. t. tigris). Meanwhile, based on the phylogenetic tree, Felinae was divided into 2 main clades: one originated 7.33 mya and included $F$. catus and $P$. bengalensis; another lineage diverged 6.97 mya with $P$. concolor, A. jubatus, and L. rufus.

\section{DISCUSSION}

In our study, we used mitochondrial genomes for phylogenetic analyses, which provided good phylogenetic resolution, spanning the entire evolutionary history of Felidae. The family was divided into 2 subfamilies, Felinae and Pantherinae, using all mitochondrial genome sequences, increasing the quality of the results. Although most of the phylogenetic tree was consistent with previous research, some clades were different compared with previous data, for example, the Panthera genus. The clouded leopard emerged first in the Panthera genus, followed by the tiger, and these clades were strongly supported by our data and previous data. The lion and leopard originated from a common ancestor after the snow leopard emerged; however, this occurred much earlier than the tiger in the phylogenetic tree constructed based on 16S rRNA and NADH binding-site-encoding gene sequences (Johnson and O'Brien, 1997). Based on fossil records, the snow leopard emerged later than the tiger. Therefore, our phylogenetic tree is more consistent with the evolution of Felidae (Johnson et al., 2006).

Combined with data for 3 fossils, the divergence times were estimated by molecular analysis, but the date obtained were inconsistent with paleogeographic data. The estimated dates were earlier than the dates suggested by the fossil records. The dates obtained from the fossils showed that the family Felidae appeared 10 mya, while the divergence time estimated by phylogenetic analysis suggested that the family appeared 14 mya (Johnson et al., 2006). Therefore, there was a large gap between the fossil record and the molecular estimation. The fossil used as the calibration point for Felidae is very rare; most fossils do not survive or have been lost. Moreover, most of Felidae comprises large animals such as the tiger, lion, and leopard, and these animals are rare in the wild; therefore, there are gaps between fossils, explaining the discrepancy between age determination using molecular estimations and fossil records (Weissengruber et al., 2002).

In conclusion, based on phylogenetic analysis using mtDNA, Felidae is divided into 2 subfamilies, Pantherinae and Felinae. All species were grouped into these 2 subfamilies, and the phylogenetic relationships were well defined. Finally, the divergence times of Felidae family members were determined by mitochondrial genome analysis. The time estimates were consistent with those estimated using other phylogenetic trees. However, the evolution of Felidae is complex and will require access to additional mitochondrial genome sequences.

\section{ACKNOWLEDGMENTS}

Research supported by the National Natural Science Foundation of China (\#30870309), and the Outstanding Ph.D. Dissertation Training Plan of the Northeast Forestry University (\#OPTP10-NEFU).

\section{REFERENCES}

Christiansen P (2008). Evolution of skull and mandible shape in cats (Carnivora: Felidae). PLoS One 3: e2807. 
Drummond AJ and Rambaut A (2007). BEAST: Bayesian evolutionary analysis by sampling trees. BMC Evol. Biol. 7: 214.

Flegontov P, Gray MW, Burger G and Lukeš J (2011). Gene fragmentation: a key to mitochondrial genome evolution in Euglenozoa? Curr. Genet. 57: 225-232.

Glazko GV and Nei M (2003). Estimation of divergence times for major lineages of primate species. Mol. Biol. Evol. 20: 424-434.

Grande C, Templado J and Zardoya R (2008). Evolution of gastropod mitochondrial genome arrangements. BMC Evol. Biol. 8: 61 .

Johnson WE and O'Brien SJ (1997). Phylogenetic reconstruction of the Felidae using 16S rRNA and NADH-5 mitochondrial genes. J. Mol. Evol. 44 (Suppl 1): S98-116.

Johnson WE, Eizirik E, Pecon-Slattery J, Murphy WJ, et al. (2006). The late Miocene radiation of modern Felidae: a genetic assessment. Science 311: 73-77.

Kan XZ, Yang JK, Li XF, Chen L, et al. (2010). Phylogeny of major lineages of galliform birds (Aves: Galliformes) based on complete mitochondrial genomes. Genet. Mol. Res. 9: 1625-1633.

Kitpipit T, Tobe SS and Linacre A (2012). The complete mitochondrial genome analysis of the tiger (Panthera tigris). Mol. Biol. Rep. 39: 5745-5754.

Krajewski C, Sipiorski JT and Anderson FE (2010). Complete mitochondrial genome sequences and the phylogeny of cranes (Gruiformes: Gruidae). The Auk 127: 440-452.

Larkin MA, Blackshields G, Brown NP, Chenna R, et al. (2007). Clustal W and Clustal X version 2.0. Bioinformatics 23: 2947-2948.

Li YP, Song W, Shi SL, Liu YQ, et al. (2010). Mitochondrial genome nucleotide substitution pattern between domesticated silkmoth, Bombyx mori, and its wild ancestors, Chinese Bombyx mandarina and Japanese Bombyx mandarina. Genet. Mol. Biol. 33: 186-189.

Luo SJ, Kim JH, Johnson WE, van der Walt J, et al. (2004). Phylogeography and genetic ancestry of tigers (Panthera tigris). PLoS Biol. 2: e442.

Mazák JH, Christiansen P and Kitchener AC (2011). Oldest known pantherine skull and evolution of the tiger. PLoS One 6: e25483.

Md-Zain BM, Mohamad M, Ernie-Muneerah MA, Ampeng A, et al. (2010). Phylogenetic relationships of Malaysian monkeys, Cercopithecidae, based on mitochondrial cytochrome c sequences. Genet. Mol. Res. 9: 1987-1996.

Morin PA, Archer FI, Foote AD, Vilstrup J, et al. (2010). Complete mitochondrial genome phylogeographic analysis of killer whales (Orcinus orca) indicates multiple species. Genome Res. 20: 908-916.

Pecon-Slattery J, Pearks Wilkerson AJ, Murphy WJ and O'Brien SJ (2004). Phylogenetic assessment of introns and SINEs within the Y chromosome using the cat family felidae as a species tree. Mol. Biol. Evol. 21: 2299-2309.

Ronquist $\mathrm{F}$ and Huelsenbeck JP (2003). MrBayes 3: Bayesian phylogenetic inference under mixed models. Bioinformatics 19: $1572-1574$.

Tamura K, Dudley J, Nei M and Kumar S (2007). MEGA4: Molecular Evolutionary Genetics Analysis (MEGA) software version 4.0. Mol. Biol. Evol. 24: 1596-1599.

Wei L, Wu X, Zhu L and Jiang Z (2011). Mitogenomic analysis of the genus Panthera. Sci. China Life Sci. 54: 917-930.

Weissengruber GE, Forstenpointner G, Peters G, Kubber-Heiss A, et al. (2002). Hyoid apparatus and pharynx in the lion (Panthera leo), jaguar (Panthera onca), tiger (Panthera tigris), cheetah (Acinonyx jubatus) and domestic cat (Felis silvestris f. catus). J. Anat. 201: 195-209.

Yang Z and Yoder AD (2003). Comparison of likelihood and Bayesian methods for estimating divergence times using multiple gene loci and calibration points, with application to a radiation of cute-looking mouse lemur species. Syst. Biol. 52: 705-716.

Yu L and Zhang YP (2005). Phylogenetic studies of pantherine cats (Felidae) based on multiple genes, with novel application of nuclear beta-fibrinogen intron 7 to carnivores. Mol. Phylogenet. Evol. 35: 483-495.

Yu L, Li QW, Ryder OA and Zhang YP (2004). Phylogenetic relationships within mammalian order Carnivora indicated by sequences of two nuclear DNA genes. Mol. Phylogenet. Evol. 33: 694-705.

Zhang W, Zhang Z, Shen F, Hou R, et al. (2006). Highly conserved D-loop-like nuclear mitochondrial sequences (Numts) in tiger (Panthera tigris). J. Genet. 85: 107-116.

Zhang W, Yue B, Wang X, Zhang X, et al. (2011). Analysis of variable sites between two complete South China tiger (Panthera tigris amoyensis) mitochondrial genomes. Mol. Biol. Rep. 38: 4257-4264. 